RELATO DE CASOS

\title{
Endometriose Colônica Simulando Câncer Colorretal - Relato de Dois Casos
}

\section{Colonic Endometriosis Simulating Colorectal Cancer: Report of 2 Cases}

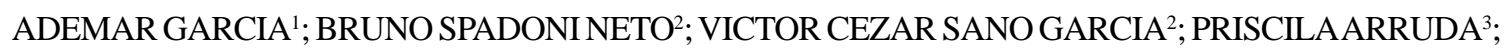 \\ DANIELALAILA GARCIA ${ }^{3}$ \\ ${ }^{1}$ Coordenador da Residência Médica de Cirurgia Geral do Hospital Geral Universitário da Universidade de Cuiabá; ${ }^{2}$ \\ Médico Residente de Cirurgia Geral do Hospital Geral Universitário da Universidade de Cuiabá; ${ }^{3}$ Acadêmica de \\ Medicina da Universidade de Cuiabá - MT - Brasil.
}

GARCIA A; SPADONI NETO B; GARCIA VCS; ARRUDA P; GARCIA DL. Endometriose Colônica Simulando Câncer Colorretal - Relato de Dois Casos Rev bras Coloproct, 2006;26(3): 316-320.

RESUMO: A Endometriose constitui doença enigmática de etiologia incerta e caracteriza-se pelo implante ectópico, extrauterino, de tecido endometrial funcionante. Sua apresentação clínica comum é de sangramento retal cíclico, associado com período menstrual e queixas como dispareunia, dismenorréia e infertilidade. Porém sua apresentação pode variar, simulando tumores colorretais e apresentando sintomas como tenesmo e sangramento retal. O diagnóstico é anatomopatológico e muitas vezes elucidado após ressecção cirúrgica. $O$ tratamento pode ser clínico ou cirúrgico, dependendo da idade, desejo de ter filhos, acometimento da lesão endometrial no trato gastrintestinal. Objetivo : relatar dois casos de Endometriose Colônica, simulando câncer colorretal, diagnosticados no serviço de Cirurgia Geral do Hospital Geral Universitário da Universidade de Cuiabá, e dissertando sobre a doença e formas de tratamento.

Descritores: Endometriose; Neoplasias Colorretais; sangramento retal.

\section{INTRODUÇÃO}

A Endometriose constitui uma doença enigmática de etiologia incerta e caracteriza-se pelo implante ectópico, extra-uterino, de tecido endometrial funcionante. Estima-se que a patologia esteja presente em $10 \%$ a $15 \%$ das mulheres em idade reprodutiva ${ }^{(5)}$. Apresenta prevalência de 4,5 a 33,3\% em mulheres submetidas a tratamento de esterilidade; 4,5 a $21,2 \%$ entre as pacientes atendidas com dor pélvica e 0 a $7,1 \%$ nas portadoras de tumoração pélvica ${ }^{(3,7)}$.

Os implantes endometriais extra-uterinos usualmente estão confinados à pelve, na região dos ovários, dos ligamentos útero-sacros e fundo de saco peritonial; porém, podem aparecer no trato gastrointestinal, trato urinário, sistema pulmonar, SNC, pele e musculatura estriada. A prevalência exata da endometriose extra-uterina não é bem conhecida. A faixa etária mais comum para início dos sintomas é de 34 a 40 anos ${ }^{(4)}$.

A endometriose intestinal é um acontecimento não raro cujos sítios de maior acometimento são o retosigmóide (73\%) e o septo reto-vaginal $(13 \%)^{(3,6,9,10,11)}$. As lesões mais freqüentes acometem as camadas serosa e muscular própria, e raramente podem ser mais profundas, comprometendo a mucosa intestinal. Os principais sintomas são dores pélvicas, constipação e/ ou diarréia recorrente, flatulência, distensão abdominal, puxo, tenesmo. Nos casos em que há acometimento da mucosa ou submucosa intestinal ocorre

Trabalho realizado no Departamento de Cirurgia Geral do Hospital Geral Universitário da Universidade de Cuiabá - Mato Grosso - Brasil.

$\overline{\text { Recebido em 14/02/2006 }}$

Aceito para publicação em 10/04/2006 
hematoquezia periódica de intensidade variável, principalmente acompanhando o período menstrual, mas às vezes ocorre sangramento anal isolado. $O$ diagnóstico e o manuseio da endometriose intestinal são difíceis, sendo fundamental o diagnóstico diferencial com outras doenças do cólon e especialmente com o câncer colo-retal, para que o tratamento correto seja adotado e procedimentos cirúrgicos extensos sejam evitados.

Apresentamos dois casos de mulheres jovens com endometriose colônica que se manifestou na forma tumoral.

\section{RELATO DO CASO 1}

SBSS, branca, 35 anos, brasileira, deu entrada no serviço de cirurgia geral com queixa de que há um ano sente dor em cólica de forte intensidade no abdome inferior, com a sensação de querer evacuar, com periodicidade de ocorrer uma a duas vezes por mês e que melhorava com uso de antiespasmódicos. Notou surgimento de uma tumoração no abdome inferior nos últimos 3 meses e que as fezes tornaram-se mais afiladas que o normal, sendo que nos últimos dois meses apresentou hematoquezia, razão que a levou a procurar o proctologista.

Como antecedente pessoal informou que fez histerectomia total mais ooforectomia direita, além de ooforectomia parcial à esquerda há três anos, por endometriose. Faz acompanhamento regular com seu ginecologista que solicitou várias vezes a realização de USG pélvica, a qual vem mostrando apenas um cisto no ovário esquerdo remanescente e confirmando ausência cirúrgica do útero e do ovário direito. Ao exame físico, observava-se a presença de uma massa palpável no hipogástrio, fixa, de consistência fibro-elástica, indolor e que, ao toque retal, comprimia extrinsecamente a parede anterior do reto, sem ulceração na mucosa, a cerca de $7 \mathrm{~cm}$ da borda anal e com presença de pequena quantidade de sangue na luva após o toque. $\mathrm{O}$ toque vaginal confirmou a presença de tal massa, fixa na parede posterior da vagina, comprimindo o reto e estendendo-se para a cavidade peritoneal.

A colonoscopia mostrou rigidez da parede colônica com mudança no pregueado mucoso, estendendo-se por cerca de $6 \mathrm{~cm}$ a partir de $9 \mathrm{~cm}$ da borda anal, e revelou também a $12 \mathrm{~cm}$ da borda anal, uma pequena formação vegetante de aspecto esbranquiçado, endurecida, sem sangramento e que foi biopsiada resultando em processo inflamatório crônico inespecífico. O enema opaco mostrava falha de enchimento na silhueta do reto por provável processo infiltrativo, numa extensão de cerca de $5 \mathrm{~cm}$, iniciando-se a $8 \mathrm{~cm}$ da borda anal. Os vários laudos de USG realizados anteriormente confirmaram apenas a presença de uma massa cística na FID com cerca de 70 $\mathrm{cm}$, sugestiva de endometrioma e a tomografia computadorizada do abdome nada acrescentou às informações já disponíveis.

Dosagens de CEA $=1,2 \mathrm{ng} / \mathrm{ml}($ normal $<5)$ e CA $125=25 \mathrm{UI} / \mathrm{ml}($ normal $<35)$.

Indicada a cirurgia que foi realizada em 21/08/ 2004 e mostrou a presença de duas massas císticas na pelve, intimamente aderidas às estruturas contíguas, porém sem fazer comunicação entre elas. Na topografia do ovário esquerdo encontramos a trompa e o ovário com uma formação cística de cerca de $50 \mathrm{~cm}$, intimamente aderida à parede anterior do reto, de forma a constituir um trajeto fistuloso (Figura 1).

Realizamos a ressecção da massa cística localizada na pelve, à direita. Também realizamos ressecção segmentar do reto-sigmóide em monobloco com o ovário e a trompa esquerda (juntamente com o cisto) e confeccionamos anastomose manual primária término-terminal, do sigmóide restante com o reto.

O exame histopatológico confirmou o diagnóstico de endometrioma nas três peças enviadas (cisto direito, cisto ovariano esquerdo e reto, onde havia um trajeto fistuloso).

A evolução pós-operatória da paciente foi excelente e ela se encontra assintomática até a presente data $(08 / 04 / 2005)$.

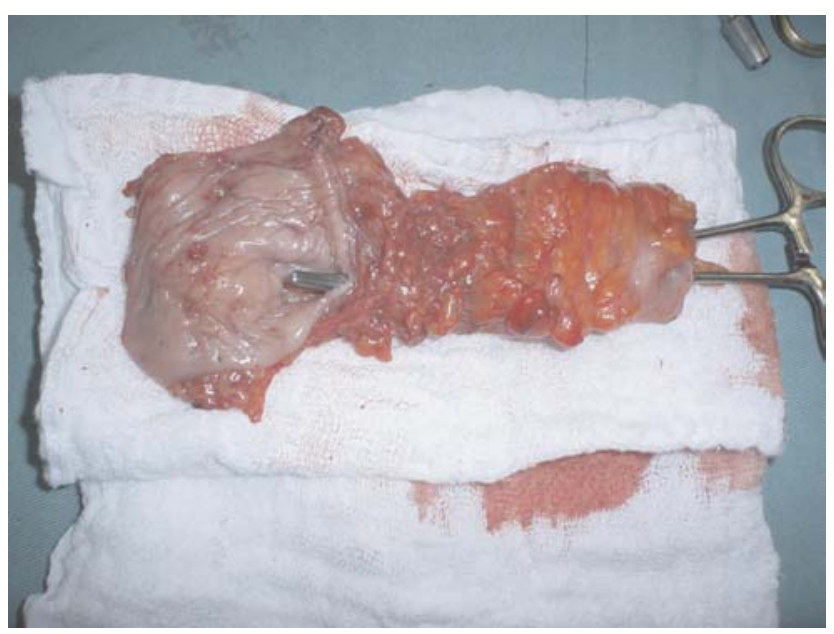

Figura 1 - Peça cirúrgica do reto mostrando o orifício fistuloso na parede anterior do reto (ponta da pinça) e alguns endometriomas. 


\section{RELATO DO CASO 2}

VRJP, 34 anos, branca, brasileira, foi atendida no serviço de cirurgia geral com queixa de dor intensa ao evacuar, de início há um ano, que ocorria no primeiro dia da menstruação, que a impedia de realizar suas atividades habituais e que pouco melhorava com uso de analgésicos.

Negava alteração do habito intestinal, mucorréia, sangramento, pus ou protusão anal, mas referia o surgimento de uma massa endurecida no abdome inferior, que estava aumentando de volume. Negava emagrecimento, mas fez tratamento de anemia no último ano. Como antecedentes pessoais relatou apenas a realização de uma cesariana há 4 anos, quando foi feita laqueadura tubária.

A palpação abdominal mostrava volumosa massa endurecida, fixa, no hipogástrio, lembrando uma gestação de 4 meses.

O toque revelava sub-estenose do reto a $9 \mathrm{~cm}$ da borda anal, que comprometia toda a circunferência do órgão e comprometia a parede vaginal posterior. $\mathrm{O}$ toque vaginal mostrava infiltração da parede posterior da vagina, sem ulceração.

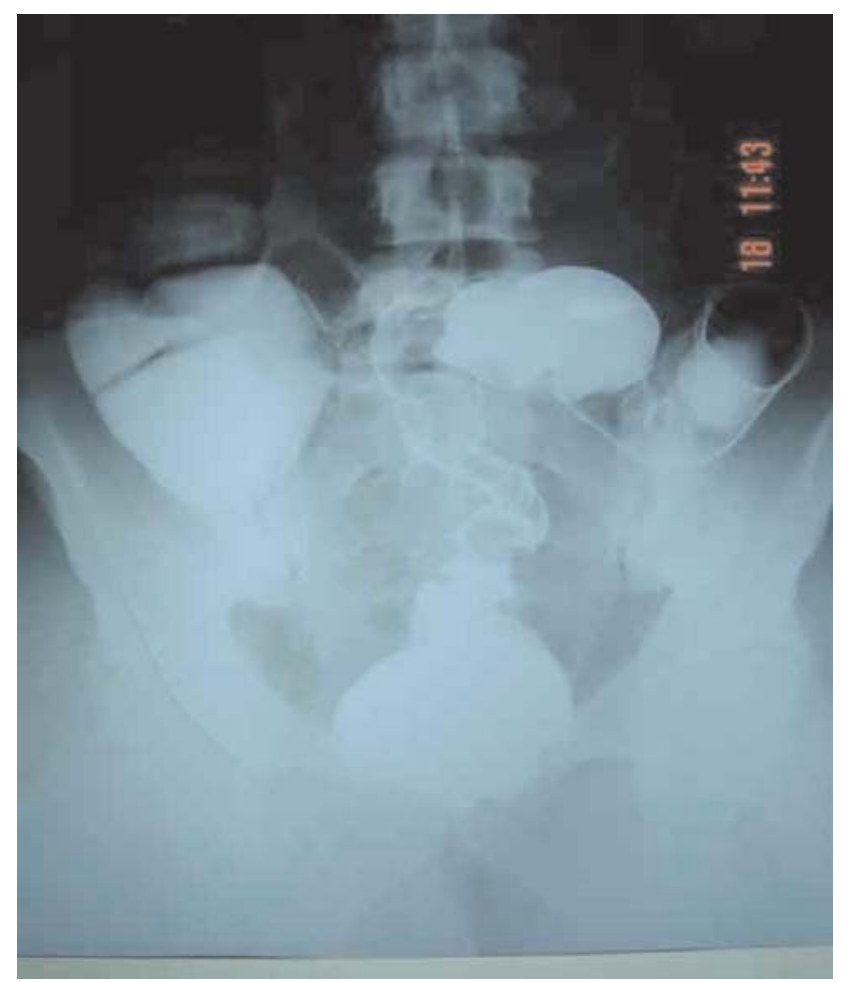

Figura 2 - O enema opaco mostrando área com aspecto de "mordida de maçã" no reto, numa extensão de cerca de $6 \mathrm{~cm}$, muito sugestiva de adenocarcinoma do reto.
Solicitados exames bioquímicos pré-operatórios, ultra-sonografia e TC do abdome, CEA, CA 125, colonoscopia e enema opaco.

A colonoscopia mostrou estenose do reto, não conseguindo ultrapassar $9 \mathrm{~cm}$ da borda anal. Foi colhido material para exame histopatológico e o resultado foi processo inflamatório crônico inespecífico. O enema opaco mostrou área com aspecto de "mordida de maçã" no reto, numa extensão de cerca de $6 \mathrm{~cm}$, muito sugestiva de adenocarcinoma retal (Figura 2).

A ultra-sonografia pélvica mostrou volume uterino de $409 \mathrm{~cm}$ com massa cística no ovário direito, sugestiva de endometrioma e a tomografia computadorizada não acrescentou maiores informações. O CEA foi de $1,0 \mathrm{ng} / \mathrm{ml}$ (normal <5) e o CA 125= $45 \mathrm{UI} / \mathrm{ml}$ (normal < 35)

A cirurgia aconteceu em 18/12/2004 quando se realizou histerectomia total, ooforectomia total bilateral e reto-sigmoidectomia segmentar anterior, mais apendicectomia. O inventário da cavidade mostrou presença de formações císticas nos dois ovários, com conteúdo achocolatado, além de volumosa hiperplasia uterina que comprimia todas as estruturas pélvicas.

A parede anterior do sigmóide e do reto intraperitoneal apresentava-se comprometida por um processo fibrótico endurecido, esbranquiçado e que causava sub-estenose da luz intestinal.

Foi feita anastomose mecânica primaria sigmóide-retal e a paciente evoluiu muito bem no pósoperatório, estando assintomática até a presente data (08/04/2005).

O exame histopatológico das peças cirúrgicas mostrou leiomioma uterino, cistos hemorrágicos de corpo lúteo nos dois ovários, trompas e apêndice cecal normais. $\mathrm{O}$ exame do segmento intestinal ressecado confirmou endometriose que comprometia a parede do sigmóide desde a serosa até a submucosa.

\section{DISCUSSÃO}

A Endometriose intestinal acomete 5\% das mulheres com endometriose pélvica ${ }^{(3,6,9,10)}$. As áreas do trato gastrointestinal mais acometidas são o reto e o cólon sigmóide em 75-90\%, sendo o íleo distal acometido em 2 a $16 \%$ e o apêndice cecal em 3 a 18\%, manifestando-se mais freqüentemente na forma de "spots" na serosa e na muscular própria, levando a fibrose e/ou estenose, sendo que a mucosa é raramente afetada ${ }^{(3,10)}$. 
A endometriose intestinal pode surgir como sangramento retal cíclico ou não, dor abdominal pélvica, constipação, obstrução intestinal e raramente pode manifestar-se na forma de perfuração ou malignização. É importante salientar que a presença da tríade clássica da endometriose (dismenorréia, dispareunia e infertilidade) contribui para aumentar a suspeita da doença intestinal concomitante, apesar de que nem sempre se manifeste em sua forma clássica.

Os exames complementares devem ser solicitados na tentativa de afastar patologias intestinais mais freqüentes e que podem apresentar os mesmos sintomas, como as neoplasias, doenças inflamatórias, colite isquêmica, colite pós-radiação, doença diverticular e até mesmo infecções. Radiologicamente, o enema baritado poderá auxiliar, mostrando lesões estenosantes, polipóides ou ambas no cólon e alguns autores recomendam como o primeiro exame a ser solicitado na suspeita de endometriose intestinal.

A colonoscopia e/ou retossigmoidoscopia deverão ser realizadas na tentativa de visualizar a característica macroscópica da lesão e de retirar fragmentos para biópsias, guiando-nos para o planejamento correto do tratamento, apesar de que a biópsia endoscópica usualmente proporciona material insuficiente para o diagnóstico patológico definitivo. Vários autores afirmam que a laparoscopia é o "padrão ouro" para firmar o diagnóstico de endometriose ${ }^{(3,6,7,9,10,11)}$.
$\mathrm{O}$ tratamento da endometriose intestinal pode ser cirúrgico ou hormonal, dependendo da idade, do desejo de manter a fertilidade, da severidade e complicações da doença. Recentemente o tratamento cirúrgico laparoscópico vem avançando estágios e provando-se factível e eficaz.

As medicações utilizadas no tratamento da endometriose são o Danazol, altas doses de progestágenos e os agonistas de GnRH, todos com eficácia semelhante. Danazol e os agonistas de GnRH possuem custo semelhante, porém o segundo é melhor tolerado.

O tratamento cirúrgico da endometriose intestinal colônica é muito discutido e controverso, porém a tendência dos últimos estudos é de realizar a ressecção segmentar da lesão com o objetivo de aliviar os sintomas e prevenir a neoplasia endometrial; a ooforectomia e o tratamento hormonal ficam indicados nas pacientes oligossintomaticas com endometriomas colorretais menos extensas ${ }^{(6,10,11)}$.

Os casos relatados anteriormente chamam a atenção pela semelhança clínica que a endometriose intestinal pode ter com as neoplasias do cólon e que seu diagnóstico é presuntivo e nem sempre pode ser afirmado anteriormente à cirurgia, assim como os exames complementares e a patologia podem ser inconclusivos, não contribuindo para diferenciar a endometriose de uma neoplasia de cólon ${ }^{(1,2,6,10)}$.

ABSTRACT: The endometriosis is an enigmatic pathology that has an uncertain etiology and is characterized by ectopic implant of functional endometrial tissue. Its most common clinical presentation is periodic abdominal pain associated with menstrual period, infertility, and complaints of dyspareunie and dysmenorrheal, but its presentation can also varies, simulating colorectal tumors by presenting symptoms like tenesm and rectal bleeding. The diagnoses is made by the anatomopathologic exam and mostly explained after surgical resection. The treatment can be done clinically or surgically, depending on the age, desire to have children and the development of endometrial lesion in the gastrointestinal tract. The aim of this work is relating two cases of colonic endometriosis simulating colorectal cancer, diagnosed at the surgical service of the Hospital Geral Universitário de Cuiabá, and dissertating about the pathology forms and its treatment.

Key words: Endometriosis; Colorectal neoplasm; rectal bleeding

\section{REFERÊNCIAS}

1. Abrão MS, Podgaec S, Filho BM, Ramos LO, Pinotti JA, de Oliveira RM. The use of biochemical markers in the diagnosis of pelvic endometriosis. Hum Reprod 1997; 12:2523-7.

2. Abrão MS, Podgaec S, Ribeiro S, Marques JA. Tratamento cirúrgico da endometriose: quando e como fazer. In: Abrão MS. Endometriose: uma visão contemporânea. Rio de Janeiro: Revinter; 2000. p. 137-47.
3. BartkowiakR,ZieniewiczK, KaminskiP, KrawczykM,Marianowiski L, Szymanska K. Diagnosis and Treatment of sigmoidal endometriosis: a case report. Med Sci Monit, 2000; 6(4): 787-0.

4. Bozdech JM. Endoscopic diagnoses of colonic endometriosis. Gastrointest Endosc 1992; 38(5) : 568-70.

5. Carvalho FM, Abrão MS, Histopatologia da endometriose: a importância da interação entre ginecologista e patologista. In: Abrão MS. Endometriose: uma visão contemporânea. Rio de Janeiro: Revinter; 2000. p. 35-44. 
6. Dimoulios P, Koutroubakis IE, Tzardi M, Antoniou P, Matalliotakis M, Kouroumalis EA. A case of sigmoid endometriosis difficult to differentiate from colon cancer. BMC Gastroenterol. 2003; 3: 18.

7. Graham B, Mazier WP. Diagnosis and management of endometriosis of the colon and rectum. Dis Colon Rectum 1998; 31(12): 952-6.

8. Olive DL, Schwartz LB. Endometriosis. N Engl J Med 1993;328:1759-69.

9. Ojeda CV, Ribeiro LC, Aguilar-Nascimento JE. Disseminação linfática na endometriose colorretal. Rev Col Bras Cir 2000;27(4):282-3.

10. Souza VCT, Baldin JA, Moreira APT. Endometriose retal Relato de caso. Rev Bras Coloproct 1996;16(4):209-11.
11. Souza JVS, Carmel APW, Silvany AM. Tratamento cirúrgico da endometriose intestinal. Rev bras Coloproct 1996;16(4):232-4.

12. Urbach DR, Reedjik M, Carole SR. Bowel resection for intestinal endometriosis. Dis Colon Rectum 1998;41(9):1158-64.

\section{Endereço para correspondência: BRUNO SPADONI NETO}

Rua Antônio Dorileo, 128 - Bairro Coophema 78.085-600- Cuiabá (MT)

Tel: (65) 9973-1770 / Fax: (65)3661- 6795

bspadonis@yahoo.com.br 